\title{
Futuribles, $n^{\circ} 267$, septembre 2001
}

\section{Alain Kimmel}

\section{OpenEdition}

\section{Journals}

Édition électronique

URL : https://journals.openedition.org/ries/1822

DOI : 10.4000/ries. 1822

ISSN : 2261-4265

\section{Éditeur}

France Education international

\section{Édition imprimée}

Date de publication : 1 septembre 2002

Pagination : 19-20

ISBN : 978-285420-554-1

ISSN : 1254-4590

\section{Référence électronique}

Alain Kimmel, «Futuribles, n² 267, septembre 2001 », Revue internationale d'éducation de Sèvres [En ligne], 30 | septembre 2002, mis en ligne le 24 novembre 2011, consulté le 05 juillet 2021. URL : http:// journals.openedition.org/ries/1822 ; DOI : https://doi.org/10.4000/ries.1822

Ce document a été généré automatiquement le 5 juillet 2021

(c) Tous droits réservés 


\title{
Futuribles, $n^{\circ} 267$, septembre 2001
}

\author{
Alain Kimmel
}

\section{RÉFÉRENCE}

Futuribles, $n^{\circ} 267$, septembre 2001

1 Cette revue mensuelle, qui se présente comme la principale revue de prospective en langue française, propose, dans ce numéro, un dossier spécial sur la prospective de l'éducation. Il rassemble quelques-unes des contributions élaborées en vue du séminaire international sur «l'École à l'horizon 2020» qui s'est tenu en 1999 au Futuroscope de Poitiers.

2 Dans son exposé introductif, intitulé «La prospective de l'éducation», Jacques Lesourne, président de Futuribles International, affirme que les évolutions des sociétés occidentales rendent nécessaire une transformation profonde des systèmes éducatifs, impliquant une plus grande autonomie des acteurs de l'éducation et une réflexion prospective débouchant sur de initiatives et des expériences.

3 L'article d'Alain Michel, coordinateur scientifique du séminaire a pour titre: «Éducation : entrer dans le XXI" siècle ». Après avoir rappelé les principaux défis lancés à l'école par les sociétés contemporaines, il analyse l'évolution de la demande sociale à son égard et réfléchit sur les connaissances et compétences communes que devront posséder les générations futures pour s'insérer dans le monde de demain.

4 Jean-Michel Saussois, consultant auprès du Centre pour la recherche et l'innovation dans l'enseignement (CERI/OCDE), s'interroge ensuite sur «la place de l'école dans l'économie en France ». Il en retrace d'abord l'histoire depuis la fin de la Seconde Guerre mondiale, à travers les "Trente Glorieuses " (Jean Fourastié) et les «Trente Piteuses » (Nicolas Baverez). La première période est celle de « l'élitisme républicain au service de la modernisation industrielle ", tandis que la seconde est celle où l'éducation doit faire "face à l'essor d'une économie de marché", avant d'être "soumise à des contradictions croissantes", notamment celle des deux conceptions de l'école (lieu de transmission d'une culture générale ou lieu de formation à la vie professionnelle). Il 
essaie ensuite de prévoir la place de l'école dans «l'économie du savoir ». Celle-ci requiert de nouvelles compétences qui, pour beaucoup, ne seront acquises et évaluées que dans un cadre professionnel. De nouveaux marchés de la connaissance émergent et l'école, qui perd le monopole de sa diffusion, n'occupe plus désormais qu'une place parmi d'autres acteurs du champ de l'éducation.

Un autre thème majeur est abordé dans ce numéro: celui des «inégalités devant l'école». Il est traité par Claude Thélot, ancien directeur de l'évaluation et de la prospective au ministère de l'Éducation nationale, qui propose une réflexion sur les inégalités géographiques et sur les inégalités sociales. Pour parvenir à une réduction de ces inégalités, il plaide en faveur d'une diversification accrue du système éducatif (notamment de ses établissements), mais en souhaitant son maintien dans un cadre national "puissant». De même, il envisage la mise en œuvre d'une politique de discrimination positive qu'il faudrait toutefois appliquer avec prudence.

Dans la même perspective, Luisa Ribolzi, professeur à l'université de Gênes, pose la question : «Quels nouveaux chemins vers l'équité ? ». À partir notamment de l'exemple italien, elle analyse les facteurs responsables de la remise en cause du concept d'équité au sein de l'école et explique pourquoi, selon elle, il s'agit d'un processus irréversible. Elle estime que le modèle égalitaire de l'école, fondé sur les principes d'unité et de neutralité du service public, est en crise et doit laisser place à une conception " équitable ", respectant les différences et la diversité des besoins. Pour atteindre cet objectif, il importe que l'institution scolaire acquière son autonomie par rapport à l'État et que ses acteurs deviennent de véritables professionnels.

7 «Quels enseignants pour 2020?» est justement l'interrogation de Françoise Cros, chercheuse à l'Institut national de recherche pédagogique (INRP). Elle y répond en esquissant le portrait-robot, que l'on pourra trouver par trop "futuriste», de l'enseignant de demain sur le plan institutionnel et structurel, sur celui de sa formation et dans sa pratique professionnelle. Il travaillera, par exemple, en « équipes mono ou pluri-catégorielles» (avec des universitaires ou des travailleurs sociaux), se formera tout au long de sa carrière et sera un " expert de l'apprentissage métacognitif », il aura un public de jeunes ou d'adultes, parlera plusieurs langues étrangères et devra travailler temporairement dans d'autres secteurs, etc.

La dernière contribution de ce dossier est celle de Francine Vaniscotte, présidente de l'Institut européen pour la promotion de l'innovation et de la culture dans l'éducation (EPICE), qui présente « les systèmes éducatifs en Europe ». Elle rappelle en premier lieu qu'il n'existe pas de modèle éducatif universel et a fortiori européen. C'est la diversité qui est la règle, avec deux conceptions dominantes : l'une qui considère le primaire et le premier cycle du secondaire de manière globale («l'école pour tous »), et l'autre qui défend le principe d'une première orientation au début du secondaire.

À partir de ce constat, elle distingue quatre types de systèmes éducatifs :

- l'école unique de 6 à 16 ans (pays scandinaves) ;

- l'école polyvalente (comprehensive school) qui propose différentes options à partir du secondaire (Royaume-Uni) ;

- l'école à filières, avec une orientation précoce et des passerelles d'un niveau à l'autre (Allemagne, Autriche, Luxembourg, Suisse, Pays-Bas, Belgique) ;

- l'école à tronc commun, proche de l'école unique (France, Espagne, Grèce, Italie, Portugal). 
10 Cette diversité s'explique par des traditions culturelles et politiques différentes, mais aussi par une forte opposition "idéologique " entre pays partisans d'un système égalitaire et pays partisans d'un système différencié.

11 Au-delà de certains aspects "prospectifs » qui peuvent laisser le lecteur sceptique, voire incrédule, ce dossier est particulièrement stimulant et, par les questions qu'il pose et les scénarios qu'il imagine, incite à la réflexion et au débat.

\section{AUTEUR}

\section{ALAIN KIMMEL}

Chargé de programme au département langue française du CIEP. 